\title{
Association between rs2107595 HDAC9 gene polymorphism and advanced carotid atherosclerosis in the Slovenian cohort
}

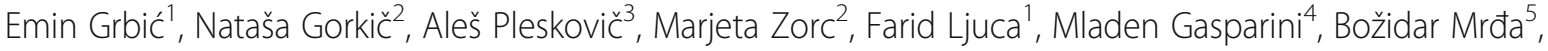
Ines Cilenšek ${ }^{6}$, Sara Mankoč ${ }^{6}$, Maciej Banach ${ }^{7,8}$, Daniel Petrovič ${ }^{2,6^{*}}$ and Zlatko Fras ${ }^{3,9,10^{*}}$

\begin{abstract}
Background: Histone deacetylase 9 (HDAC9) plays an important role in transcriptional regulation, cell cycle progression and developmental events; moreover, it has been investigated as a candidate gene in a number of conditions, including the onset and progression of atherosclerosis. We hypothesized that the rs2107595 HDAC9 gene polymorphism may be associated with advanced carotid artery disease in a Slovenian cohort. We also investigated the effect of this polymorphism on HDAC9 receptor expression in the internal carotid artery (ICA) specimens obtained by endarterectomy.

Methods: This case-control study enrolled 619 unrelated Slovenian patients: 311 patients with ICA stenosis > 75\% as the study group and 308 patients with ICA stenosis $<50 \%$ as the control group. Patient laboratory and clinical data were obtained from the medical records. The rs2107595 polymorphisms were genotyped using TaqMan SNP Genotyping assay. HDAC9 expression was assessed by immunohistochemistry in 30 ICA specimens from patients with ICA atherosclerosis $>75 \%$, and the numerical areal density of HDAC9 positive cells was calculated.

Results: The occurrence of advanced ICA atherosclerosis in the Slovenian cohort was 3.81 times higher in the codominant genetic model $(\mathrm{OR}=3.81,95 \% \mathrm{Cl}=1.06-13.77, p=0.04)$, and 3.10 times higher in the recessive genetic model $(\mathrm{OR}=3.10,95 \% \mathrm{Cl}=1.16-8.27, p=0.02)$. In addition, the A allele of rs2107595 was associated with increased HDAC9 expression in the ICA specimens obtained by endarterectomy.

Conclusions: We observed a significant association between the AA genotype of rs2107595 with the advanced carotid artery disease in our Slovenian cohort, indicating that this polymorphism may be a genetic risk factor for ICA atherosclerosis.

Keywords: Histone deacetylase 9 (HDAC9) gene polymorphism, Atherosclerosis, Carotid atherosclerosis, Advanced carotid atherosclerosis
\end{abstract}

\section{Introduction}

Atherosclerosis is a chronic inflammatory disease characterized by the accumulation of fat, cholesterol, calcium and other substances from the blood into the inner layers of arterial blood vessels of various size. Atherosclerosis

\footnotetext{
* Correspondence: daniel.petrovic@mf.uni-lj.si; zlatkofras63@gmail.com ${ }^{2}$ International Center for Cardiovascular Diseases MC Medicor d.d, Izola, Slovenia

${ }^{3}$ Department of Cardiology, Division of Medicine, University Medical Centre of Ljubljana, Ljubljana, Slovenia

Full list of author information is available at the end of the article
}

related vascular disease (ASCVD) can lead to different clinical manifestations. These are related to the vascular bed mostly affected, and among the most dangerous are the atherosclerotic complications within the coronary or carotid arteries, manifested as either the heart attack or stroke, respectively [1].

Carotid atherosclerosis is among the major causes of stroke and transient ischemic attacks (TIA). An increase in the stenosis of the internal carotid artery diameter of

(c) The Author(s). 2020 Open Access This article is licensed under a Creative Commons Attribution 4.0 International License, which permits use, sharing, adaptation, distribution and reproduction in any medium or format, as long as you give appropriate credit to the original author(s) and the source, provide a link to the Creative Commons licence, and indicate if changes were made. The images or other third party material in this article are included in the article's Creative Commons licence, unless indicated otherwise in a credit line to the material. If material is not included in the article's Creative Commons licence and your intended use is not permitted by statutory regulation or exceeds the permitted use, you will need to obtain permission directly from the copyright holder. To view a copy of this licence, visit http://creativecommons.org/licenses/by/4.0/. The Creative Commons Public Domain Dedication waiver (http://creativecommons.org/publicdomain/zero/1.0/) applies to the data made available in this article, unless otherwise stated in a credit line to the data. 
more than $50 \%$ is associated with about $15 \%$ of cases of ischemic stroke (IS) [2].

Stroke rates differ around the world; for example, from 9 per 100,000 in Qatar and 138 per 100,000 people in Russia. The average stroke death rate in Europe is 96 per 100,000 people, while in North America it is 19 per 100,000 people per year [3]. It has also been observed that the mortality rate increases with age and varies in highly and poorly developed economies [4]. As the number of patients with ASCVD increases with age, so do the effects it has on the quality of life of people with the disease. Therefore, more research is needed to prevent and slow the progression of atherosclerosis.

It is generally accepted that epigenetic and genetic factors play an important role in the onset and progression of ASCVD [5-10]. An important role in epigenetic activity plays the HDAC9 enzyme, encoded by the Histone Deacetylase 9 (HDAC9) gene, which affects the chromosomal structure and performs histone deacetylation by inhibiting transcription [11]. HDAC9 is a protein coding gene that belongs to the histone deacetylase superfamily, class IIA. It is located on chromosome 7p21.1, $915.4 \mathrm{~kb}$ in size and encodes a protein responsible for histone deacetylation [12]. The polymorphisms of $H D A C 9$ gene affect the acetylation and deacetylation processes of histones and thus further cause the activation or inactivation of certain genes $[12,13]$. The most common polymorphism of the HDAC9 gene is rs 2107595 , located in the 3 ' region of the HDAC9 gene.

So far, HDAC9 has been reported to affect several aspects of the pathogenesis of atherosclerosis, i.e. cholesterol efflux, platelet aggregation, interleukin 6 (IL-6) signaling, macrophage function, inflammation progression and vascular calcification [6, 11, 12, 14]. Moreover, several studies have found an association between the rs2107595 polymorphism of the HDAC9 gene and the onset and progression of carotid atherosclerosis [12], ischemic and hemorrhagic stroke $[15,16]$, large vessel atherosclerotic stroke (LVAS) [14], and atherosclerotic coronary artery disease [17]. However, the data on the rs2107595 polymorphism and its association with the progression of carotid atherosclerosis are still limited.

In this study, we aimed to investigate the association between the rs 2107595 polymorphism of the HDAC9 gene and advanced carotid artery disease in a Slovenian cohort. The second aim was to investigate the effect of the above-mentioned SNPs on the expression of the HDAC9 gene within the endarterectomy specimens obtained by surgery.

\section{Materials and methods}

\section{Patients}

In a present case-control study we enrolled 619 unrelated Caucasians, 311 consecutive patients with advanced carotid atherosclerosis (internal carotid artery (ICA) stenosis $>75 \%$ ) and history of stroke/transitory ischemic attack (cases), and 308 control subjects. The control group encompassed consecutive subjects examined at the outpatient cardiology department for routinely planned cardiovascular risk assessment, so we include subjects of both genders without symptomatic carotid artery disease, i.e. either without any kind of ultrasound detectable atherosclerotic changes or subjects with mild atherosclerotic changes, however, the grade (percentage) of stenosis of common carotid artery (CCA) or ICA had to be hemodynamically nonsignificant, i.e. less than $50 \%$. The cases enrolled into the study were revascularized, either by performing carotid surgery or with carotid stent implantation. Cases and control subjects were recruited from 3 Slovenian Health Care facilities, Medical Centre Medicor d.d. Ljubljana, Izola General Hospital and University Clinical Center Maribor, in the period from 2010 to 2019.

The degree of stenosis was determined by duplex vascular ultrasound examination and, if necessary for clinical purposes / reasons, also computed tomography (CT) angiography of the carotid arteries was applied. Ultrasound examinations and CT angiographies were performed by six specialists (three cardiologists and three radiologists) from the aforementioned institutions. The vascular ultrasound examinations consist of quantitative measurements of intima media thickness (IMT), the presence, type, and thickness of atherosclerotic plaques, blood flow rate, and assessment of the narrowing rate of CCA, ICA, and external carotid artery (ECA). The IMT on the left and right sides of CCA, ICA, and ECA were presented as the arithmetic mean of the three measurements $[18,19]$.

General information and history of risk behaviours, as well as anthropometric, clinical and laboratory parameters measured, such as gender, age, systolic and diastolic blood pressure, smoking status, alcohol, glucose, physical activity level, body mass index (BMI), cholesterol, high density lipoprotein cholesterol (HDL-C), low density lipoprotein cholesterol (LDL-C), triglycerides, high-sensitivity Creactive protein (CRP), waist circumference, glycated hemoglobin (HbA1c), coronary artery disease (CAD), myocardial infarction (MI), duration of arterial hypertension $(\mathrm{AH})$, and duration of diabetes mellitus (T2DM), were taken from cautiously taken health medical records.

To avoid the potential influence of confounding factors, patients with carotid artery stenosis of nonatherosclerotic origin, patients with neck tumor, patients with aortic arc stenosis and right artery subclavian stenosis, as well as patients with incomplete data, were not enrolled in the study. The study was approved by the National Medical Ethics Committee and was designed in accordance with the Declaration of Helsinki. After informative consent was signed, a detailed interview and physical examination were performed. 


\section{Genotyping}

Deoxyribonucleic acid (DNA) was isolated from peripheral blood leukocytes in the laboratory of the Institute of Histology and Embryology, Faculty of Medicine, University of Ljubljana. Isolation of genomic DNA was performed on a QIAcube apparatus (Qiagen $\mathrm{GmbH}$, Hilden, Germany) according to the "V3" protocol, using a commercially available QIAamp DNA Blood Mini Kit (250) (Qiagen GmbH, Hilden, Germany), which included five different reagents (AL buffer, 96\% ethanol, AW1 buffer, AW2 buffer, AE buffer) and an appropriate amount of protease (Qiagen $\mathrm{GmbH}$, Hilden, Germany) $(285 \mu \mathrm{L} / 200 \mu \mathrm{L}$ blood). Following all the steps from the manufacturer's instructions, 3-12 $\mu \mathrm{g}$ of genomic DNA, i.e. $30-40 \mathrm{ng} / \mu \mathrm{L}$, was isolated from $200 \mu \mathrm{L}$ of blood.

The rs2107595 polymorphism was genotyped using TaqMan SNP Genotyping assay (Applied Biosystems, Foster City, CA, USA), according to the manufacturer's recommendations. Genotyping was carried out in both groups (cases and controls).

\section{Immunohistochemistry}

Immunohistochemistry (IHE) staining was performed on 30 endarterectomy specimens obtained by surgery. Formalin-xedpara n-embedded (FFPE) tissue sections of ICA were used for hematoxylin-eosin (HE) staining. Consecutive sections of $5 \mu \mathrm{m}$ tissue were cut and then mounted and dried on glass slides from each paraffin block. Tissues were deparaffinized and dehydrated in graded alcohol solutions. The detection of HDAC9positive cells was performed with the Novo Link Max Polymer Detection System (Leica Biosystems Newcastle Ltd., United Kingdom) following the manufacturer's instructions. The slides were incubated with antiHDAC9 monoclonal antibodies (TermoFisher, USA), diluted 1:100, overnight at $4{ }^{\circ} \mathrm{C}$. Placental tissue was used as a positive, and tonsils as a negative control. The cells were defined as HDAC9-positive/negative. The area with HDAC9-positive cells was manually marked and numerical areal density of HDAC9-positive cells was calculated (the number of positive cells per $\mathrm{mm}^{2}$ ) $[20,21]$.

\section{Statistical analysis}

Statistical analysis was performed using the SPSS, ver. 26.0, software for MS Windows (IBM SPSS, New York, USA). Normal distribution of data was checked using the Shapiro-Wilk test. Normally distributed continuous clinical data are presented as a mean $\pm \mathrm{SD}$, while nonnormally distributed data (continuous variables) are presented as a median and interquartile range. Categorical variables are presented as numbers and percentage of patients affected. Normally distributed continuous clinical data were compared with an unpaired Student-t test, nonnormally distributed continuous data were compared with
Mann - Whitney U test. Discrete variables were compared by chi-square test. All variables where significant differences were obtained by univariate analysis $(p<$ $0.05)$ were analyzed by logistic regression analysis. The deviation from Hardy-Weinberg equilibrium (HWE) was assessed by chi-square goodness of fit test.

\section{Results}

History data, general anthropometric and laboratory characteristics of cases (subjects with ICA stenosis > $75 \%$ ) and controls (subjects with no carotid atherosclerosis or ICA stenosis $<50 \%$ ) are presented in Table 1. There were no significant differences between the groups with respect to BMI, HDL-C levels, triglycerides, HbA1c, coronary artery calcium (CAC) score, presence and duration of $\mathrm{T} 2 \mathrm{DM}$, and duration of $\mathrm{AH}$. On the other hand, statistically significant differences were found between the groups in the following parameters: age $(p<0.001)$, gender $(p<0.001)$, excessive alcohol consumption $(p<0.001)$, smoking $(p<0.001)$, waist circumference $(p<0.001)$, systolic blood pressure (SBP) $(p<0.001)$, diastolic blood pressure (DBP) $(p<0.04)$, total cholesterol $(p<0.001)$ and LDL-C levels $(p<0.001)$, hs-CRP $(p<0.001)$, AH $(p<0.001)$, CAD $(p<0.001)$ and MI $(p<0.033)$. Patients from the case group (with stenosis $>75 \%$ ) were older, had higher waist circumference, and greater alcohol and tobacco smoking use than in comparison to the control group. Also, patients from the case group had higher SBP, DBP, higher total cholesterol and LDL-C levels, lower HDL-C levels, as well as significantly higher hs-CRP values compared to the control group.

The distribution of genotype and allele frequency of the rs2107595 polymorphism of the HDAC9 gene in the subjects from the case and control groups is shown in Table 2. By univariate analysis we found significant differences in genotype $(p<0.02)$ and allele $(p<0.01)$ frequencies between the case group and controls. Genotype distribution did not deviate significantly from Hardy-Weinberg equilibrium.

We used logistic regression analysis to assess whether rs21007595 polymorphism was independently associated with the progression of atherosclerosis after adjusting for age, waist circumference, tobacco smoking, SBP, DBP, total cholesterol, LDL-C levels, and hs-CRP. The results indicate the existence of a statistically significant association in two genetic models, codominant (OR 3.81; CI 1.06-13.77; $p=0.04$ ), and recessive (OR 3.10; CI 1.16-8.27; $p=0.02$ ) (Table 3).

Within the endarterectomy sequesters obtained from surgically treated patients with advanced ICA, a statistically significantly higher numerical areal density of HDAC9-positive cells was found in subjects with the A 
Table 1 Anthropometric, clinical and laboratory characteristics of of the groups of cases and controls

\begin{tabular}{|c|c|c|c|}
\hline Characteristics & Cases (311) & Controls (308) & $P$ value \\
\hline Number & 311 & 308 & \\
\hline Age (years) & $70.5 \pm 8.5$ & $62.9 \pm 11.6$ & $<0.001$ \\
\hline BMI $\left(\mathrm{kg} / \mathrm{m}^{2}\right)$ & $28.3 \pm 4.4$ & $28.7 \pm 4.3$ & 0.42 \\
\hline Waist (cm) & $102.2 \pm 12.5$ & $96.7 \pm 14.7$ & $<0.001$ \\
\hline Male sex (\%) & $208(67.2)$ & $148(47.7)$ & $<0.001$ \\
\hline Systolic blood pressure (mm Hg) & $144.9 \pm 20.0$ & $136.6 \pm 18.0$ & $<0.001$ \\
\hline Diastolic blood pressure $(\mathrm{mm} \mathrm{Hg})$ & $80.2 \pm 10.6$ & $83.0 \pm 13.4$ & 0.04 \\
\hline Blood glucose level (mmol/L) & $5.9(5.2-7.6)$ & $5.3(4.8-6.6)$ & $<0.001$ \\
\hline History of arterial hypertension (\%) & $286(91.5)$ & $206(66.9)$ & $<0.001$ \\
\hline Tobacco smoking (\%) & $81(26.0)$ & $40(13.0)$ & $<0.001$ \\
\hline Total cholesterol (mmol/L) & $4.5 \pm 1.3$ & $5.1 \pm 1.2$ & $<0.001$ \\
\hline LDL cholesterol (mmol/L) & $2.4(1.9-3.2)$ & $2.9(2.2-3.8)$ & $<0.001$ \\
\hline HDL cholesterol (mmol/L) & $1.2 \pm 0.3$ & $1.4 \pm 0.4$ & 0.14 \\
\hline Triglycerides (mmol/L) & $1.4(1.0-1.9)$ & $1.4(1.0-2.0)$ & 0,61 \\
\hline $\mathrm{HbA}_{1 c}(\%)^{\mathrm{a}}$ & $7.8 \pm 1.3$ & $7.2 \pm 1.5$ & 0,19 \\
\hline hs-CRP (mg/L) & $3.0(2.5-8.0)$ & $1.5(0.8-3.5)$ & $<0.001$ \\
\hline Diabetes mellitus (\%) & $109(34.5)$ & 95 (30.9) & 0.39 \\
\hline Myocardial infarction (\%) & $44(13.8)$ & $28(8.6)$ & 0.03 \\
\hline
\end{tabular}

allele in comparison with HDAC9 homozygotes (wild type) $\left(755 \pm 235 / \mathrm{mm}^{2}\right.$ versus $\left.392 \pm 153 / \mathrm{mm}^{2} ; p<0.001\right)$.

\section{Discussion}

In the present study we investigated the association between the rs2107595 of the HDAC9 gene and advanced carotid atherosclerosis in a Slovenian cohort of subjects. Logistic regression analysis clearly showed a significant tendency of the carriers of the rs2107595 polymorphism towards an increased risk for the progression of carotid atherosclerosis in two genetic models (co-dominant and recessive). Anyway, the results obtained and showed above need further to be discussed, mainly from the aspect of their comparability with the

Table 2 Genotype and allele distribution of rs2107595 in cases and controls

\begin{tabular}{llll}
\hline & Cases (311) & Controls (308) & $p$ value \\
\hline AA (MAF) & $20(6.4 \%)$ & $7(2.3 \%)$ & \\
AG & $105(33.8 \%)$ & $94(30.5 \%)$ & $\mathbf{0 . 0 2}$ \\
GG & $186(59.8 \%)$ & $207(67.2 \%)$ & \\
A allele (\%) & $145(23.3 \%)$ & $108(17.5 \%)$ & $\mathbf{0 . 0 1}$ \\
G allele (\%) & $477(76.7 \%)$ & $508(82.5 \%)$ & \\
HWE & 0.33 & 0.33 & \\
\hline
\end{tabular}

HWE Hardy-Weinberg equilibrium to date published results of other similar research, but also from the point of view of their potential use in clinical practice, as well as the limitations of the present study.

Markus et al. [16] investigated the association of the rs2107595-HDAC9 gene with LVAS, IMT, and asymptomatic carotid plaque in community samples. Also, they investigated the expression of HDAC9 in the cerebral and major arteries. They found an association of the rs2107595 polymorphism with IMT $(p=0.0018)$ as well as the presence of carotid plaque $(p=0.0022)$. Immunohistochemical analysis revealed the presence of HDAC9 in both endothelial and vascular smooth muscle cells. Also, Azghandi et al. [17] suggested that the risk allele of the HDAC9 gene exerts its effects through the increased expression of HDAC9. They also highlighted the association of the HDAC9 gene with the onset of atherosclerosis, heart attack and stroke [22]. Furthermore, Shroff et al. [14] investigated the influence of the rs2107595 HDAC9 gene on the differences in leukocyte gene expression in patients with LVAS. LVAS patients with the risk allele and controls without it showed a statistically significant difference in the expression of HDAC9 $(p<0.05)$. Also, they suggested an association of rs2107595 of the HDAC9 with peripheral immune, lipid, and coagulation pathways in patients with LVAS [14]. These studies show that the role of the HDAC9 gene 
Table 3 Logistic regression analysis adjusted for different confounders (age, waist, sex, SBP, DBP, history of hypertension, tobacco smoking, total cholesterol, LDL cholesterol, hs-CRP, MI, Blood Glucose level) according to the co-dominant, dominant and recessive genetic models

\begin{tabular}{|c|c|c|c|}
\hline & CASES/CTRLS & OR $(95 \% \mathrm{Cl})$ & $p \dagger$ \\
\hline \multicolumn{4}{|l|}{ CODOMINANT (rs2107595) } \\
\hline AA vs. GG*(reference) & $20 / 7$ vs. $186 / 207$ & $3.81(1.06-13.77)$ & 0.04 \\
\hline AG vs. GG*(reference) & $105 / 94$ vs. $186 / 207$ & $1.21(0.74-1.97)$ & 0.45 \\
\hline \multicolumn{4}{|l|}{ DOMINANT (rs2107595) } \\
\hline$[A A+A G]$ vs. GG*(reference) & $125 / 101$ vs. $186 / 207$ & $1.35(0.85-2.16)$ & 0.26 \\
\hline \multicolumn{4}{|l|}{ RECESSIVE (rs2107595) } \\
\hline AA vs. $[A G+G G]^{*}$ reference & 20/7 vs. 291/301 & $3.10(1.16-8.27)$ & 0.02 \\
\hline
\end{tabular}

$O R$ odds ratio, $\mathrm{Cl}$ confidence interval

p† values - adjusted for age, waist, sex, SBP, DBP, history of AH, tobacco smoking, total cholesterol, LDL-C, hs-CRP, MI, blood glucose level

and its rs2107595 polymorphism is one of the major causes of atherosclerosis and associated diseases. Specifically, HDAC9 is at a key position in the pathophysiological pathways leading to the formation of atherosclerotic plaque. Our findings are in line with this study and also suggest an association of rs2107595 polymorphism with the progression of carotid atherosclerosis, as well as an increased risk of manifest cerebrovascular events in Slovenian subjects.

Opposite to our study, Su et al. [23] in the Chinese Han population found no association of the rs2107595 polymorphism and the IS onset in any of the genetic models (all $p>0.05$ ) [23]. Comparing these results to the European population we have to consider the potential influence of different environmental and epigenetic factors. We did not find any other papers which would had published the results opposite to ours.

Most studies which found the high expression of HDAC9, highlight its role in the onset and progression of atherosclerosis, large vessel atherosclerotic stroke, increased risk of stroke and heart attack, etc. Therefore, there is also a growing body of research highlighting the importance of using selective and non-selective HDAC9 inhibitors in the treatment and prevention of atherosclerosis, heart attack, and stroke. With this regard, the study performed by Brookes et al. [22] emphasizes the importance of HDAC9 in the pathogenesis of stroke, as well as the possible positive effect of using sodium valproate (as a non-selective HDAC9 inhibitor) in reducing the recurrence rate of stroke $(p=0.002)$ [19]. HDAC9-selective inhibitors are still in the experimental testing stages, and the agent A4291 has the best forecasts so far [24].

However, we have to report also several important limitations related to our study. First of all, the number of subjects included was relatively small what influences the validity and reliability of the results obtained. Second, the study was conducted on an ethnically homogeneous population of a small society what significantly limits its generalizability. Furthermore, we processed only one
HDAC9 gene polymorphism, so we cannot exclude the potential influence of other important polymorphisms. In general, it is widely known that there are many potential SNPs that might significantly influence the risk of the ASCVD development. Here we have to acknowledge the already discussed and published fact that currently only the multifactorial/multiSNPs approach (with the consequent potential of construction of reliable and validates polygenic risk scores) can be widely recommended [25-27].

Anyway, the results of our study suggest the potential of the use of rs2107595 polymorphisms determination in order of refined assessment of the risk for advanced atherosclerotic carotid disease in clinical settings, not only in patients with already established ASCVD in different vascular beds, e.g. in patients with coronary or peripheral arterial disease, but also in subjects presented with no manifest disease. Furthermore, we can assume the potential applicability also in close relatives of patients with advanced carotid disease, with the aim of potential adjustments of the level of applied preventive therapies. The confirmation of the existence of the rs2107595 polymorphism can guide the treatment towards much more aggressive approach, with more strict advice and monitoring with regard to the therapeutic lifestyle changes and even lower target values of classical ASCVD major risk factors set (e.g. to insist in adjustment of the diet to decrease the systemic proinflammatory state, as well as earlier to achieve preferably recommended blood pressure and the lowest possible levels of atherogenic blood lipids, primarily of LDL-C). However, we need more research on larger samples to assure the necessary validity of the predictive value of the existing rs2107595 polymorphism to be used as a true ASCVD risk modifier.

In conclusion, we demonstrated an association of the rs2107595 polymorphism of the HDAC9 gene with the advanced carotid artery disease in Slovenian cohort. We speculate that the mechanism of action of the HDAC9 rs2107595 polymorphism might be via the increased 
expression of HDAC9 in atherosclerotic plaques. Further research with a larger sample should elucidate the role of rs2107595 in the onset and progression of ASCVD as such and the potential serious clinical consequences that accompany it, such as ischemic stroke.

\section{Abbreviations}

AH: Arterial hypertension; ASCVD: Atherosclerosis related cardiovascular disease; BMI: Body mass index; CAC: Coronary artery calcium; CAD: Coronary artery disease; CCA: Common carotid artery; CT: Computed tomography; DBP: Diastolic blood pressure; DNA: Deoxyribonucleic acid; ECA: External carotid artery; FFPE: Formalin-xedpara n-embedded; HbA1c: Glycated hemoglobin; HDAC9: Histone deacetylase 9; HDL-C: High density lipoprotein cholesterol; hs-CRP: high-sensitivity C-reactive protein; HWE: Hardy-Weinberg equilibrium; ICA: Internal carotid artery; IHE: Immunohistochemistry; IL6: Interleukin 6; IMT: Intima media thickness; IS: Ischemic stroke; LDL-C: Low density lipoprotein cholesterol; LVAS: Large vessel atherosclerotic stroke; MI: Myocardial infarction; rs2107595: rs2107595 single nucleotide polymorphism; SBP: Systolic blood pressure; SNP: Single nucleotide polymorphism; T2DM: Diabetes mellitus type 2; TIA: Transitory ischemic attack

\section{Acknowledgements}

The authors thank Mrs. Visam Bajt, BA, for revising the English. Moreover, we would like to thank Mrs. Marjeta Kuhar and Miha Krkovič, BA for genetical analysis.

\section{Authors' contributions}

Emin Grbić, MD study design, manuscript draft writing, reviewing and approval Nataša Gorkič, MD patients evaluation / data acquisition, manuscript draft approval Aleš Pleskovič, MD, PhD patients evaluation / data acquisition, manuscript draft approval Marjeta Zorc, MD, PhD patients evaluation / data acquisition, manuscript draft approval Farid Ljuca, MD, PhD study design, manuscript draft approval Mladen Gasparini, MD, PhD patients evaluation / data acquisition, manuscript draft approval Božidar Mrđa, MD patients evaluation / data acquisition, manuscript draft approval Ines Cilenšek, PhD study design, statistical analysis, manuscript draft approval Sara Mankoč, MD, PhD study design, statistical analysis, manuscript draft approval Maciej Banach, MD, PhD manuscript draft reviewing and approval Daniel Petrovič, MD, PhD conception \& study design, data analysis, manuscript draft writing, reviewing and approval Zlatko FRAS, PhD, MD conception \& study design, data analysis, manuscript draft writing, reviewing and approval. The authors read and approved the final manuscript.

\section{Funding}

The study was supported in part by the funding from the Slovenian Research Agency, programme (code P3-0326), and the internal research and development funds of the University Medical Centre of Ljubljana (code 20140272)

\section{Availability of data and materials}

The datasets used and/or analysed during the current study are available from the corresponding author on reasonable request. All the data presented (and according sources) within the paper are fully available for a review.

\section{Ethics approval and consent to participate}

(1) The study was approved by the National Medical Ethics Committee of the Republic of Slovenia, and was designed in accordance with the Declaration of Helsinki.

(2) Every patient was included only after the Informative Consent (developed in accordance with the standards of the National Medical Ethics Committee of the Republic of Slovenia) with the detailed explanation of the study was signed.

\section{Consent for publication}

All the members of the Authors' Group gave their signed consent and agree with the publication of the manuscript in the submitted form.

\section{Competing interests}

The authors declare that they have no competing interests in connection with the research published in the present paper.

\section{Author details}

'Department of Physiology, Faculty of Medicine, University of Tuzla, Tuzla, Bosnia and Herzegovina. ${ }^{2}$ International Center for Cardiovascular Diseases MC Medicor d.d, Izola, Slovenia. ${ }^{3}$ Department of Cardiology, Division of Medicine, University Medical Centre of Ljubljana, Ljubljana, Slovenia.

${ }^{4}$ Department of Vascular Surgery, General Hospital Izola, Izola, Slovenia. ${ }^{5}$ Department of Vascular Surgery, University Medical Centre Maribor, Maribor, Slovenia. ${ }^{6}$ Institute of Histology and Embryology, Faculty of Medicine, University of Ljubljana, Vrazov trg 2, SI-1000 Ljubljana, Slovenia.

${ }^{7}$ Cardiovascular Research Centre, University of Zielona-Gora, Zielona Gora, Poland. ${ }^{8}$ Polish Mother's Memorial Hospital Research Institute (PMMHRI), Lodz, Poland. ${ }^{9}$ Division of Medicine, Centre for Preventive Cardiology, Division of Medicine, University Medical Centre Ljubljana, Zaloška cesta 7, SI-1525 Ljubljana, Slovenia. ${ }^{10}$ Chair of Internal Medicine, Medical Faculty, University of Ljubljana, Ljubljana, Slovenia.

Received: 18 January 2020 Accepted: 31 March 2020

Published online: 13 April 2020

\section{References}

1. Atherosclerosis. National Heart, Lung, and Blood Institute (NHLBI). Accessed 04.01.2020 at the URL: https://www.nhlbi.nih.gov/health-topics/ atherosclerosis.

2. Elhfnawy AM, Volkmann J, Schliesser M, Fluri F. Symptomatic vs. Asymptomatic 20-40\% Internal Carotid Artery Stenosis: Does the Plaque Size Matter? Front Neurol. 2019;10:960.

3. Krishnamurthi RV, Feigin VL, Forouzanfar MH, Mensah GA, Connor M, Bennett DA, et al. Global and regional burden of first-ever ischaemic and haemorrhagic stroke during 1990-2010: findings from the global burden of disease study 2010. Lancet Glob Health. 2013;1:e259-81.

4. Herrington W, Lacey B, Sherliker P, Armitage J, Lewington S. Epidemiology of atherosclerosis and the potential to reduce the global burden of Atherothrombotic disease. Circ Res. 2016;118:535-46.

5. Prasher D, Greenway SC, Singh RB. The impact of epigenetics on cardiovascular disease. Biochem Cell Biol. 2020;98(1):12-22.

6. Lusis AJ. Genetics of atherosclerosis. Trends Genet. 2012;28:267-75.

7. Khyzha N, Alizada A, Wilson MD, Fish JE. Epigenetics of atherosclerosis: emerging mechanisms and methods. Trends Mol Med. 2017:23:332-47.

8. Totomoch-Serra A, Muñoz ML, Burgueño J, Revilla-Monsalve MC, Diaz-Badillo A. Association of common polymorphisms in the VEGFA and SIRT1 genes with type 2 diabetes-related traits in Mexicans. Arch Med Sci. 2018;14:1361-73.

9. Hajighasemi S, Mahdavi Gorabi A, Bianconi V, Pirro M, Banach M, Ahmadi Tafti $\mathrm{H}$, et al. A review of gene- and cell-based therapies for familial hypercholesterolemia. Pharmacol Res. 2019:143:119-32.

10. Dziemidowicz M, Bonda TA, Litvinovich S, Taranta A, Winnicka MM, Kamińsk KA. The role of interleukin-6 in intracellular signal transduction after chronic $\beta$-adrenergic stimulation in mouse myocardium. Arch Med Sci. 2019;15: 1565-75.

11. Smith JD. 2014. New role for histone deacetylase 9 in atherosclerosis and inflammation. Arterioscler Thromb Vasc Biol. 2014;34:1798-9.

12. Petrie K, Guidez F, Howell L, Healy L, Waxman S, Greaves M, Zelent A. The histone deacetylase 9 gene encodes multiple protein isoforms. J Biol Chem. 2003;278:16059-72.

13. Han Z, Dong X, Zhang C, Wu Y, Yuan Z, Wang X. Polymorphism of HDAC9 gene is associated with increased risk of acute coronary syndrome in Chinese Han population. Biomed Res Int. 2016;2016:1-6.

14. Shroff N, Ander BP, Zhan X, Stamova B, Liu D, Hull H. HDAC9 polymorphism alters blood gene expression in patients with large vessel atherosclerotic stroke. Transl Stroke Res. 2019;10:19-25.

15. Chauhan G, Debette S. Genetic risk factors for ischemic and hemorrhagic stroke. Curr Cardiol Rep. 2016;18:124.

16. Markus HS, Mäkelä K-M, Bevan S, Raitoharju E, Oksala N, Bi JC, et al. Evidence HDAC9 genetic variant associated with ischemic stroke increases risk via promoting carotid atherosclerosis. Stroke. 2013;44:220-5.

17. Azghandi S, Prell C, van der Laan SW, Schneider M, Malik R, Berer K, et al. Deficiency of the stroke relevant HDAC9 gene attenuates atherosclerosis in accord with allele-specific effects at 7p21.1. Stroke. 2015;46:197-202. 
18. Nikolajević-Starčević J, Pleskovič A, Santl Letonja M, Jenko Pražnikar Z, Petrovič D. Polymorphisms $+45 T>G$ and $+276 G>T$ of the adiponectin gene does not affect plasma adiponectin level and carotid intima-media thickness in patients with diabetes mellitus type 2. Int Angiol. 2014;33:434-40.

19. Paternoster L, Martinez-Gonzalez NA, Charleton R, Chung M, Lewis S, Sudlow CLM. Genetic effects on carotid intima-media thickness: systematic assessment and meta-analyses of candidate gene polymorphisms studied in more than 5000 subjects. Circ Cardiovasc Genet. 2010;3:15-21.

20. Urbancic M, Stunf S, Milutinovic AZ, Petrovic D, Petrovic MG. Epiretinal membrane inflammatory cells density might reflect the activity of proliferative diabetic retinopathy. Invest Ophthalmol Vis Sci. 2014;55:8576-82.

21. Weibel ER. Practical methods for biological morphometry. In: Stereological methods. London: Academic; 1979

22. Brookes RL, Crichton S, Wolfe CDA, Yi Q, Li L, Hankey GJ, et al. Sodium valproate, a histone Deacetylase inhibitor, is associated with reduced stroke risk after previous ischemic stroke or transient ischemic attack. Stroke. 2018;49:54-61.

23. Su L, Shen T, Liang B, Xie J, Tan J, Chen Q, et al. Association of GWASsupported loci rs2107595 in HDAC9 gene with ischemic stroke in southern Han Chinese. Gene. 2015;570:282-7.

24. Hsu K-C, Liu C-Y, Lin TE, Hsieh J-H, Sung T-Y, Tseng H-J, et al. Novel class Ilaselective histone Deacetylase inhibitors discovered using an in Silico virtual screening approach. Sci Rep. 2017;7:3228.

25. Moulin P, Dufour R, Averna M, Arca M, Cefalù AB, Noto D, et al. Identification and diagnosis of patients with familial chylomicronaemia syndrome (FCS): expert panel recommendations and proposal of an "FCS score". Atherosclerosis. 2018;275:265-72.

26. Sathiyakumar V, Kapoor K, Jones SR, Banach M, Martin SS, Toth PP. Novel therapeutic targets for managing dyslipidemia. Trends Pharmacol Sci. 2018; 39:733-47.

27. Khera AV, Chaffin M, Zekavat SM, Collins RL, Roselli C, Natarajan P, et al. Whole-genome sequencing to characterize monogenic and polygenic contributions in patients hospitalized with early-onset myocardial infarction. Circulation. 2019:139:1593-602.

\section{Publisher's Note}

Springer Nature remains neutral with regard to jurisdictional claims in published maps and institutional affiliations.

Ready to submit your research? Choose BMC and benefit from:

- fast, convenient online submission

- thorough peer review by experienced researchers in your field

- rapid publication on acceptance

- support for research data, including large and complex data types

- gold Open Access which fosters wider collaboration and increased citations

- maximum visibility for your research: over $100 \mathrm{M}$ website views per year

At $\mathrm{BMC}$, research is always in progress.

Learn more biomedcentral.com/submissions 